Proceeding Series of the Brazilian Society of Computational and Applied Mathematics

\title{
Análise de Erro do Compressive Sensing para Dados Sísmicos
}

\section{Éberton da Silva Marinho'}

Programa de Pós-Graduação em Ciências e Engenharia do Petróleo, UFRN, Natal, Brasil

\section{Francisco Iranildo Ferreira do Nascimento Gomes ${ }^{2}$}

Programa de Pós-Graduação em Ciências e Engenharia do Petróleo, UFRN, Natal, Brasil

Tiago Cavalcanti da Rocha

Programa de Pós-Graduação em Ciências e Engenharia do Petróleo, UFRN, Natal, Brasil

\section{Gilberto Corso ${ }^{4}$}

Departamento de Biofísica e Farmacologia, Centro de Biociências, UFRN, Natal, Brasil

\section{Liacir dos Santos Lucena}

Departamento de Física Teórica e Experimental, UFRN, Natal, Brasil

International Center for Complex Systems, UFRN, Natal, Brasil

Programa de Pós-Graduação em Ciência e Engenharia do Petróleo, UFRN, Natal, Brasil

Resumo. O Bayesian Compressive Sensing é aplicado a um sismograma real usando várias amostragens e duas bases Wavelets: Daubechies 6 e Symlet 9. A distribuição de erro do sinal recuperado pelo Compressive Sensing tende a ser normal para baixas amostragens e não normal, muito assimétrica e leptocúrtica, para altas amostragens.

Palavras-chave. Compressive Sensing, Bayesian Compressive Sensing, Wavelets, Sismogramas, Análise de erro.

\section{Introdução}

A aquisição e processamento de sinais sísmicos têm uma grande importância na indústria do petróleo. Tais atividades são primordiais para um melhor entendimento e

\footnotetext{
1 ebertonsm@gmail.com, eberton.marinho@ifrn.edu.br

2 ironrdc@gmail.com

3 supremotiago@gmail.com

4 gfcorso@gmail.com

5 liacir.lucena@gmail.com
} 
predição de camadas e estruturas formadoras do subsolo. Elas comporão o estudo de viabilidade de exploração de uma determinada região juntamente com outras análises $[8$, 9].

A técnica do Compressive Sensing (CS) $[1,2,3]$ tem se mostrado útil na tarefa de reduzir o volume de dados na aquisição de um sinal. O CS contorna um dos maiores limitadores da amostragem que é o teorema de Shannon-Nyquist. Este teorema afirma que para se restaurar um sinal com precisão, faz-se necessária uma taxa de amostragem que tenha pelo menos o dobro da frequência mais alta dos dados.

Apesar do sucesso da CS, sua utilizacão na área do petróleo ainda é recente. Neste trabalho realizamos, sucintamente, um estudo do erro na recuperação de sinais sísmicos usando o CS para várias taxas de amostragem do sinal e com o emprego de duas Wavelets [4, $5,7]$ distintas como bases. De fato, fizemos uma exploração heurística do comportamento de várias Wavelets, sendo que a Daubechies 6 e a Symlet 9 se mostraram as mais promissoras para nossas análises. Além disto, estudamos o algoritmo Bayesian Compressive Sensing (BCS) $[6,10]$ e o empregamos nesta trabalho. Para analisar o erro da reconstrução do sinal estudamos sua distribuição. Em especial, testamos a normalidade, a simetria e verificamos o quanto a distribuição pode ser mesocúrtica.

\section{Metodologia}

\subsection{Dados sísmicos}

A reflexão sísmica é uma das técnicas mais utilizadas no levantamento geológico na área de exploração de petróleo. Cada traço sísmico é o resultado da captação das informações refletidas pelo sistema de camadas terrestres, sendo que, a junção de vários traços sísmicos dá origem a um sismograma. Os dados sísmicos aqui utilizados são compostos por 33 traços que foram retirados do Capítulo 4 de [8] e encontram-se disponíveis em (http://www.morganclaypool.com/doi/pdfplus/10.2200/S00384ED1V01 Y201109SPR010).

Todos os scripts utilizados foram criados no ambiente de programação MATLAB (http://www.mathworks.com/).

\subsection{Compressive Sensing}

Considere um sinal $x[i]$, como por exemplo, uma série temporal com $N$ elementos. Em seguida usamos uma base do tipo Wavelet, $\Psi=\left[\psi_{1}\left|\psi_{2}\right| \ldots \mid \psi_{N}\right], N \times N$, para $\psi_{i}$ um vetor coluna. Desta forma, o sinal original $x$ pode ser expresso como

$$
x=\sum_{i=1}^{N} s_{i} \psi_{i} \quad \text { ou } \quad x=\Psi s
$$

onde $s$ é um vetor coluna de coeficientes reescrito como segue: $s_{i}=\left\langle x, \psi_{i}\right\rangle=\psi_{i}^{T} x$. Podese ver que $s$ e $x$ são representações equivalentes de um mesmo sinal, porém $x$ se encontra no domínio do tempo, enquanto $s_{i}$ está no domínio de $\Psi$. O sinal $x$ será do tipo $K$ esparso se ele puder ser escrito como uma combinação linear de apenas $K$ vetores, isto é, $K<<$. 
Para simularmos o processo de aquisição comprimida do sinal, vamos supor um processo de medição linear geral $\Phi=\left\{\phi_{j}\right\}_{j=1}^{M}$. Este processo computa $M<N$ produtos internos entre o sinal original $x$ e uma base de vetores, como em $y_{j}=\left\langle x, \phi_{j}\right\rangle$, onde $M \sim K$. Com auxílio de (1) pode-se escrever

$$
y=\Phi x+e=\Phi \Psi s+e=\Theta s+e
$$

onde $\Theta=\Phi \Psi$ é uma matriz $M \times N$. Ademais, a quantidade $e$ aqui será o erro. Destacamos que no processo do CS a medição é não adaptativa, ou seja, a matriz $\Theta$ não depende de $x[1,2,4]$.

\subsection{Bayesian Compressive Sensing}

Uma abordagem Bayesiana $[6,10]$ pode ser aplicada ao problema de recuperação, onde o sinal $s$ desconhecido é associado a uma probabilidade $p(s \mid \gamma)$, chamada de distribuição a priori, que modela nosso conhecimento sobre a natureza de $s$. O y é modelado como um conjunto de observações resultantes de um processo aleatório com distribuição condicional $p(y \mid s, \beta)$, onde $\beta=1 / \sigma^{2}$ é o inverso da variância do ruído. Estas distribuições dependem do modelo de parâmetros $\gamma$ e $\beta$, que são hiperparâmetros, e distribuições a priori adicionais, chamadas hiperprioris.

A modelagem proposta constitui uma forma hierárquica de três estágios. Os primeiros dois estágios desta priori hierárquica resultam em uma distribuição de Laplace $p(s \mid \lambda)$, e o último estágio é incorporado para calcular $\lambda$. Na Figura 1, em (a), temos o sinal original (em azul) do traço 17 e, em (b), a comparação com a recuperação pelo método BCS para $30 \%$ do sinal original utilizando a Wavelet Daubechies 6 (sinal recuperado em vermelho).

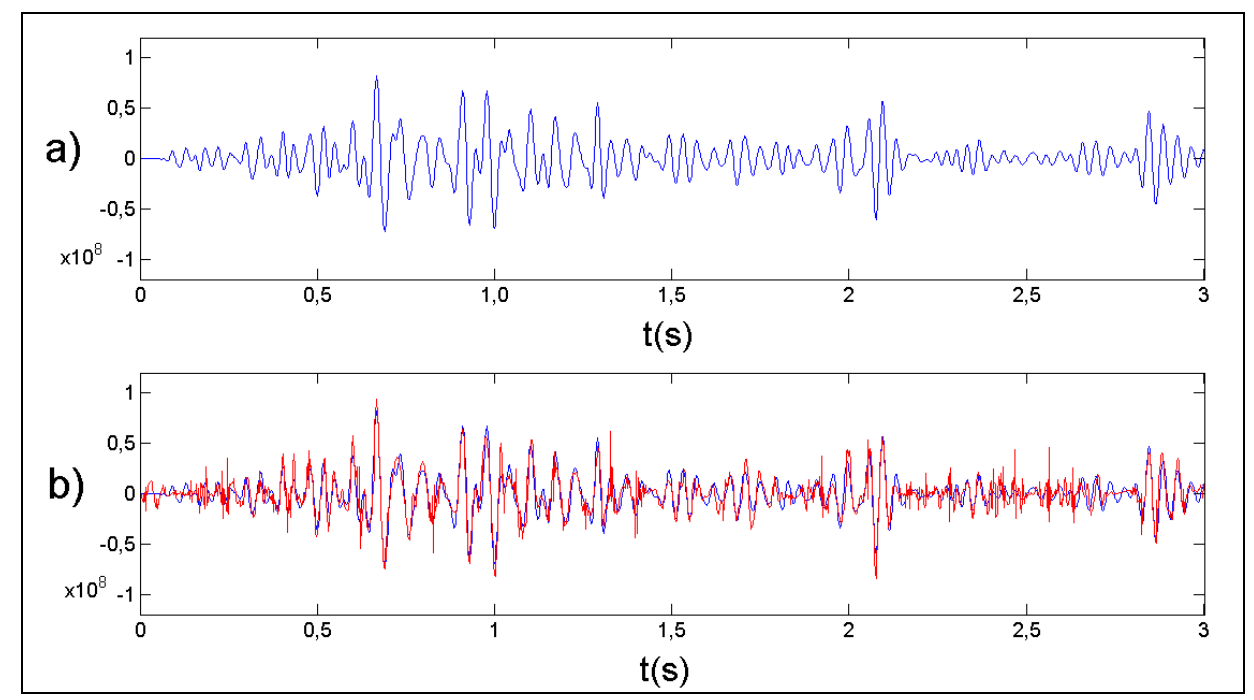

Figura 1: Exemplo de um traço sísmico. Em a), a série original de dados do traço número $17 \mathrm{com} 1501$ pontos. Em b), a superposição do traço sísmico original (em azul) e o recuperado (em vermelho) com amostragem de 30\%, utilizando a Wavelet Daubechies 6. 


\section{Análises dos Histogramas do Erro}

Após procedermos a amostragem e a recuperação para 1000 execuções de cada traço utilizando o método BCS com vários parâmetros (cinco amostragens e duas Wavelets), calculamos o erro relativo. A matriz de sensoriamento que usamos neste trabalho foi aleatória gaussiana com média nula e desvio padrão inversamente proporcional ao número de amostras. Em seguida, para cada traço, criamos histogramas do erro relativo, como os apresentados na Figura 2. Para a execução do algoritmo BCS foi usada a função BCS_fast_rvm disponível na biblioteca SparseLab versão 2.1 que se encontram no site https://sparselab.stanford.edu/.

Com os dados dos histogramas, calculamos as médias e desvios padrões da recuperação do sinais com cada uma das 5 amostras utilizando as Wavelets Daubechies 6 e Symlet 9, apresentados na Tabela 1. Em seguida, para cada traço, computamos os testes de normalidade (pelo método Kolmogorov-Smirnov), de simetria e de curtose, apresentados na Tabela 2.

As Tabelas 1 e 2 indicam, de uma forma resumida, três tendências: (i) o erro relativo diminui com o aumento da amostragem; (ii) amostragens muito altas (50\%) levam a distribuições não normais, ainda mais assimétricas à direita e leptocúrticas; (iii) parece haver uma transição na resposta do Comprensive Sensing quando a taxa de aquisição se situa entre 40 e 50\%. Ressaltamos também que o desvio da curtose observado foi para tendência leptocúrtica (os erros se tornam mais concentrados junto a média) quando a distribuição deixa de ser normal. Observou-se também que o desvio da assimetria sempre foi para a direita, ou seja, a recuperação do Compressive Sensing tem maior probabilidade de produzir sinais recuperados com grande erro relativo. Acrescenta-se que qualitativamente os resultados se mostraram semelhantes para as duas Wavelets estudadas.

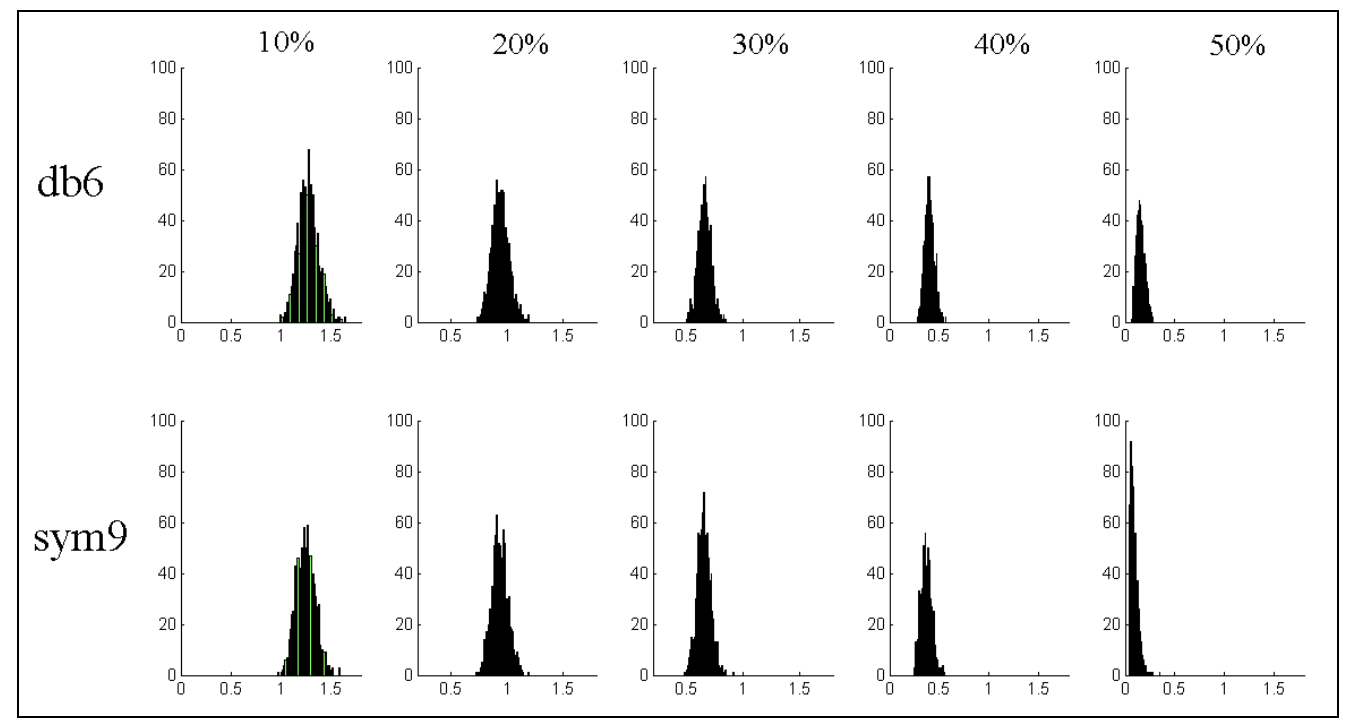

Figura 2: Histogramas do erro relativo da recuperação do traço de número 17 através do BCS para várias amostragens. O eixo da abscissa representa o erro relativo e o eixo da ordenada a frequência absoluta. 
Tabela 1: Medições das médias $(\mu)$ e desvio padrões $(\sigma)$ para o conjunto de todos os traços. Nesta tabela são indicados os valores para as taxas de amostragem e duas Wavelets.

\begin{tabular}{|c|c|c|c|c|c|}
\hline$\mu \pm \sigma$ & $10 \%$ & $20 \%$ & $30 \%$ & $40 \%$ & $50 \%$ \\
\hline db6 & $1,28 \pm 0,12$ & $0,96 \pm 0,13$ & $0,67 \pm 0,12$ & $0,37 \pm 0,10$ & $0,09 \pm 0,05$ \\
\hline sym9 & $1,33 \pm 0,15$ & $0,94 \pm 0,13$ & $0,65 \pm 0,12$ & $0,36 \pm 0,09$ & $0,07 \pm 0,05$ \\
\hline
\end{tabular}

Tabela 2: Tabela quantitativa para os testes de normalidade, simetria e curtose da recuperação do sismograma pelo método BCS.

\begin{tabular}{|c|l|c|c|c|c|c|}
\hline & Testes & $10 \%$ & $20 \%$ & $30 \%$ & $40 \%$ & $50 \%$ \\
\hline \multirow{4}{*}{ db6 } & Normal por Kolmogorov-Smirnov & 32 & 29 & 33 & 28 & 0 \\
\cline { 2 - 7 } & Simetria & 8 & 9 & 5 & 15 & 0 \\
\cline { 2 - 7 } & Inclinação à Direita & 25 & 24 & 28 & 18 & 33 \\
\cline { 2 - 7 } & Curtose (Mesocúrtico) & 27 & 20 & 30 & 27 & 1 \\
\cline { 2 - 7 } & Leptocúrtico & 6 & 13 & 3 & 6 & 32 \\
\hline \multirow{5}{*}{ sym9 } & Normal por Kolmogorov-Smirnov & 28 & 27 & 33 & 29 & 1 \\
\cline { 2 - 7 } & Simetria & 10 & 6 & 6 & 14 & 1 \\
\cline { 2 - 7 } & Inclinação à Direita & 23 & 27 & 27 & 18 & 32 \\
\cline { 2 - 7 } & Curtose (Mesocúrtico) & 28 & 26 & 30 & 26 & 2 \\
\cline { 2 - 7 } & Leptocúrtico & 5 & 7 & 3 & 7 & 31 \\
\hline
\end{tabular}

\section{Conclusões e Trabalhos Futuros}

Aplicamos a técnica do Comprensive Sensing sobre um sismograma real usando duas bases Wavelets: Daubechies 6 e Symlet 9 . O estudo da distribuição dos erros é importante na indústria do petróleo, porque ajuda a identificar falhas em imagens sísmicas que podem levar a problemas de interpretação geofísicas. Uma distribuição de erro que se desvia da normalidade é potencialmente perigosa em engenharia, pois a presença de assimetria marcante no sinal gera caudas longas e a possibilidade de falsos resultados oriundos de dados muito distantes da média.

Uma análise sobre o erro do sinal recuperado mostra que, como esperado, o erro diminui com o aumento da amostragem do sinal, tanto do ponto de vista da média do erro, quanto da sua flutuação em em torno da média. Porém, amostragens altas (50\%) levam a distribuições de erro não normais, ainda mais assimétricas e não mesocúrticas. Este resultado sugere um ponto frágil do Comprensive Sensing que deve ser explorado em mais detalhes. Por fim uma outra questão também se mostrou de interesse. Os resultados indicam uma transição na distribuição de erro quando se varia a taxa de aquisição neste sistema. Existe um ponto entre 40 e $50 \%$ onde parece haver uma descontinuidade na resposta do Comprensive Sensing. Uma analise em torno desta transição é um dos objetos de futuras pesquisas, bem como a realização de testes semelhantes com outras Wavelets e outros métodos de recuperação, com ou sem filtros. 


\section{Referências}

[1] R. G. Baraniuk, Compressive sensing, IEEE Signal Processing Magazine, vol. 24, 118 121, (2007), DOI: 10.1109/MPS.2007.4286571.

[2] E. Candès, J. Romberg and T. Tao, Robust uncertainty principles: Exact signal reconstruction from highly incomplete frequency information, IEEE Trans. Information Theory, vol. 52, no. 2, pp. 489-509, (2006), DOI: 10.1109/TIT.2005.862083.

[3] D. L. Donoho, Compressed sensing, IEEE Trans. Information Theory, vol. 52, no. 4, pp. 1289-1306, (2006), DOI: 10.1109/TIT.2006.871582.

[4] M. Duarte, M. Wakin and R. Baraniuk, Wavelet-domain Compressive Signal Reconstruction Using a Hidden Markov Tree Model, IEEE Int. Conf. on Acoustics, Speech, and Signal Processing(ICASSP), Las Vegas, Nevada, (2008), DOI: 10.1109/ICASSP.2008.4518815.

[5] L. He and L. Carin, Exploiting Structure in Wavelet-Based Bayesian Compressive Sensing,IEEE Trans. Signal Processing, vol. 57, no. 9, pp.3488-3497, (2009), DOI: 10.1109/TSP.2009.2022003.

[6] S. Ji, Y. Xue and L. Carin, Bayesian compressive sensing, IEEE Trans. Signal Processing, vol. 56, no. 6, pp. 2346-2356, (2008), DOI: 10.1109/TSP.2009.2022003.

[7] S. Mallat, A wavelet tour of signal processing, 2nd ed., Academic Press, (1998), DOI: $10.1109 \% 2 \mathrm{~F} 34.192463$.

[8] W. A. Mousa and A. A. Al-Shuhail, Processing of Seismic Reflection Data Using MATLAB, Synthesis Lectures on Signal Processing, Morgan \& Claypool Publishers, (2011), DOI: 10.2200/S00384ED1V01Y201109SPR010.

[9] E. Onajite, Seismic Data Analysis Techniques in Hydrocarbon Exploration, 1st ed., Elsevier (2013), ISBN-10: 0124200230

[10] M. W. Seeger and H. Nickisch, Compressed sensing and Bayesian experimental design, in ICML, pp. 912-919. (2008), DOI: 10.1145/1390156.1390271. 